\title{
Señales físico químicas involucradas en la búsqueda de hospederos y en la inducción de picadura por mosquitos
}

José Luis Torres-Estrada, M en C, Dr en C, ${ }^{(1)}$ Mario H Rodríguez, MC, Dr en Filosofía. ${ }^{(2)}$

\section{Torres-Estrada JL, Rodríguez MH. Señales físico químicas involucradas en la búsqueda de hospederos y en la inducción de picadura por mosquitos.} Salud Publica Mex 2003;45:497-505. El texto completo en inglés de este artículo está disponible en: http://www.insp.mx/salud/index.html

\section{Resumen}

Las hembras de los mosquitos vectores de enfermedades utilizan señales físicas y químicas para localizar su fuente de alimentación sanguínea en hospederos vertebrados. Los mosquitos zoofílicos responden preferentemente al $\mathrm{CO}_{2}$ y al octenol liberados en la respiración y excreciones, mientras que los mosquitos antropofílicos responden al ácido láctico y a una variedad de compuestos del sudor. Estos compuestos son modificados por microrganismos saprófitos de las glándulas sebáceas de la piel. 0 tros factores presentes en las viviendas contribuyen a la integración de microsistemas constituidos por olores caracteristicos, que explican los diferentes niveles de atracción de mosquitos y la focalización de la transmisión del paludismo a una porción de casas en localidades de áreas endémicas. La identificación de estos atrayentes químicos y sus moléculas recepto ras en mosquitos puede ser utilizada como complemento de nuevos métodos para la vigilancia epidemio lógica, para atraer a los mosquitos a trampas de colecta o para incrementar su contacto con insecticidas usados en su control, así como en la manipulación genética para desviar las picaduras de los mosquitos hacia otros hospederos vertebrados. El texto completo en inglés de este artículo está disponible en: http://www.insp.mx/salud/index.html

Palabras clave: mosquitos; atrayentes; emanaciones humanas; malaria; México

\author{
Torres-Estrada JL, Rodríguez MH. \\ Physic-chemical signals involved \\ in host localization and induction \\ of disease vector mosquito bites. \\ Salud Publica Mex 2003;45:497-505. \\ The English version of this paper \\ is available at: http://www.insp.mx/salud/index.html
}

\begin{abstract}
A bstract
Abstract. Disease vector female mosquitoes respond to physic-chemical signals to localize vertebrate hosts for blood meals. Zoophylic mosquitoes preferentially respond to $\mathrm{CO}_{2}$ and octenol released in the breath and bodily fluids, while anthropo phylic mosquitoes respond to lactic acid and a variety of sweat compounds. These compounds are modified by sapro phytic microorganisms in the skin sebaceo us glands. 0 ther factors present in human dwellings contribute to the integration of microsystems with characteristic odors that have different attraction for mosquitoes, explaining the focalization of malaria transmission in few households in endemic areas. The identification of the chemical attractants and their molecular receptors could be used to complement new methods to attract mosquitoes to traps during epidemiological surveys, to increase their contact with insecticides in control interventions, and for genetic manipulation to divert mosquito bites towards other animal populations. The English version of this paper is available at: http://www.insp.mx/salud/index.html
\end{abstract}

Key words: mosquitoes; attractants; human emanations; malaria; Mexico

(1) Centro de Investigación de Paludismo, Instituto N acional de Salud Pública (IN SP), Tapachula, Chiapas, México.

(2) Centro de Investigación sobre Enfermedades Infecciosas, IN SP, Cuernavaca, Morelos, México.

Fecha de recibido: 7 de febrero de 2003 - Fecha de aprobado: 4 de septiembre de 2003

Solicitud de sobretiros: Dr. José Luis Torres Estrada. Centro de Investigación de Paludismo, Instituto N acional de Salud Pública. 4a A venida norte y 19 poniente, colonia Centro, 30700 Tapachula, C hiapas, México.

Correo electrónico: jltorres@ correo.insp.mx 
L a alimentación sanguínea es el momento culminante de la interacción entre artrópodos y hospederos vertebrados. Esta interacción ha dado como resultado la aparición de ciclos de vida complejos en varios organismos, que al desarrollarse en los artrópodos los convierte en vectores, y al desarrollarse en vertebrados producen enfermedades. Desde el punto de vista epidemiológico, los comportamientos más importantes que determinan la capacidad de los mosquitos vectores de transmitir enfermedades son la búsqueda de sangre y los conducentes a la puesta de sus huevos. Estas actividades son indispensables para la reproducción de estas especies ${ }^{1}$ y cobran importancia epidemiológica, porque son determinantes del contacto humano-vector, indispensable para la transmisión de organismos patógenos.

Los comportamientos de búsqueda de hospederos y de sitios de oviposición, aunque independientes entre sí, ocurren en forma coordinada. El comportamiento de búsqueda de un sitio para depositar los huevos no puede ocurrir al mismo tiempo que cuando la señal fisiológica para el comportamiento de búsqueda de hospedero es liberada. Así, en el caso de hembras nulíparas, la búsqueda de sangre tiene prioridad sobre cualquier otro comportamiento y solamente es inhibido por la distensión abdominal que ocurre después de alimentarse.

En estudios epidemiológicos, la intensidad del contacto humano-vector en una población determinada se infiere por medio de la medición del índice de sangre humana, un indicador de su preferencia alimenticia $^{2-5}$ y por la medición del ciclo gonotrófico, un indicador de la frecuencia de alimentación, ${ }^{6-10}$ puesto que los mosquitos requieren de al menos una alimentación sanguínea para producir cada lote de huevos. Si bien la información en estos indicadores es útil para evaluar la capacidad vectorial de diversas poblaciones de mosquitos, no permite identificar los factores que determinan la intensidad de contacto humanovector. La identificación de estos factores podría ser de utilidad para establecer las condiciones que determinan los patrones de transmisión de patógenos y para el desarrollo de nuevas estrategias de control.

\section{Factores determinantes del comportamiento}

En términos generales, los procesos fisiológicos más importantes en mosquitos están mediados por olores. La respuesta de los mosquitos a los olores ocurre dentro de un marco de referencia dado por factores externos (lluvia, viento, humedad y temperatura) y factores internos (edad, sexo, estado fisiológico y preferencia alimenticia) que influyen en el comportamiento de selección de la fuente de olor. Dentro de este comportamiento, la activación y orientación están supeditadas a las condiciones del medio (velocidad del viento, temperatura e intensidad de la luz) mientras que el aterrizaje sobre el hospedero está influenciado por las características específicas de la fuente de olor (figura 1).

Si bien el estado fisiológico de los mosquitos determina su comportamiento, la expresión de éste es influida por estímulos físicos del ambiente y es mediada por señales químicas. Estas señales emanan de sitios con características adecuadas para la oviposición y de los posibles hospederos vertebrados que son posibles fuentes de sangre. ${ }^{11}$ Presentamos aquí una revisión de los atrayentes identificados que median el comportamiento de búsqueda de hospedero de los principales vectores del paludismo en el mundo.

\section{Señales que induce la búsqueda y picadura de hospederos}

En mosquitos, la inducción del comportamiento de búsqueda de hospederos es mediada por estímulos físicos y químicos. ${ }^{12}$ Los estímulos físicos (visuales) juegan un papel importante en la activación del comportamiento de búsqueda y localización de un hospedero a larga distancia (ejemplo: contraste e intensidad de luces, movimiento). ${ }^{13}$ Los estímulos visuales son el activador del vuelo de cualquiera de los comportamientos antes señalados; un insecto en busca de hospederos responde a niveles de luz del entorno ambiental y de la fuente misma (intensidad de luz), pero los vuelos apetitivos a larga distancia son dirigidos por blancos visuales. ${ }^{13}$ Mientras que a distancias cortas, las señales químicas ayudan a los mosquitos a identificar el flujo (pluma) de olor (estímulos químicos) que los orientan hacia el hospedero. ${ }^{14}$

La respuesta de los mosquitos hembra a estos estímulos depende de su preferencia por el tipo de hospedero, así como de su edad y estado fisiológico (nutrición, digestión y grado de desarrollo de los huevos en los ovarios). ${ }^{15}$ Los estímulos químicos provocan la respuesta anemotáctica (orientación en contra del viento siguiendo un gradiente de olor) de búsqueda del hospedero y desencadenan la estímulación final para que se lleven a cabo el piquete y la alimentación (figura. 1).

\section{Participación del $\mathrm{CO}_{2}$}

La percepción de la presencia de un posible hospedero se inicia con la detección del $\mathrm{CO}_{2}$ expedido por un mamífero vertebrado. Esta percepción provoca que el 


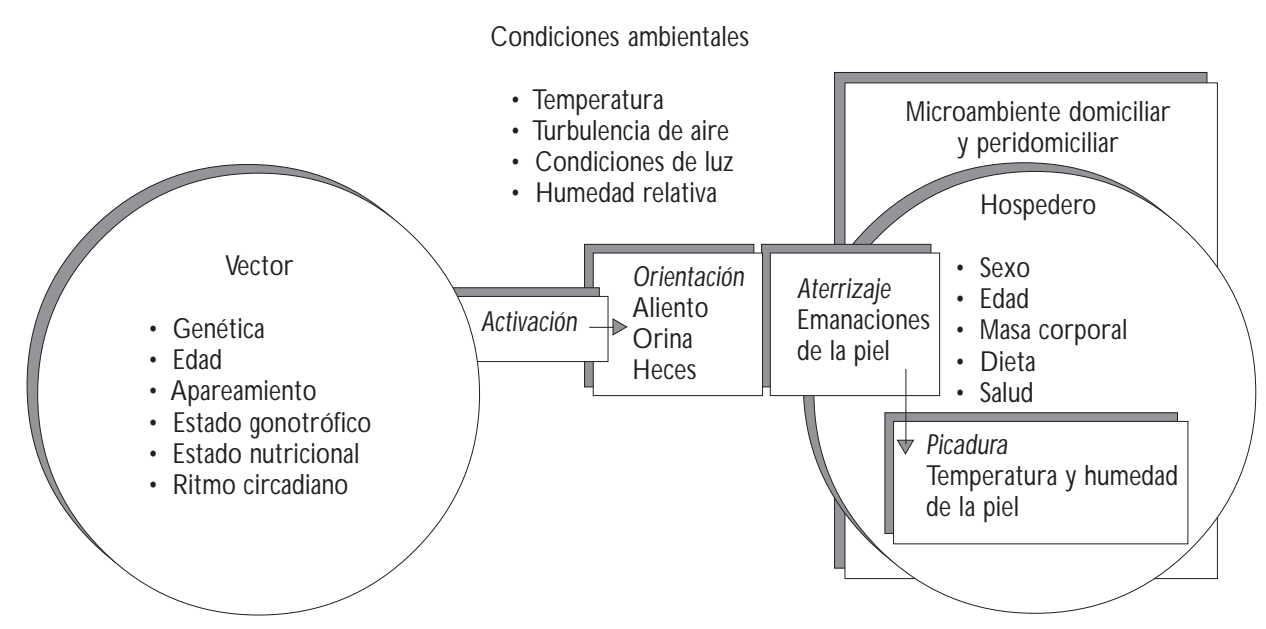

Figura 1. Representación diagramática de los factores Que intervienen en la búsqueda de hospederos VerteBRADOS POR MOSQUITOS. LA BÚSQUEDA DE UNA COMIDA DE SANGRE ESTÁ INFLUIDA POR FACTORES PROPIOS DEL MOSQUITO (VECTOR), EL POSIBLE HOSPEDERO VERTEBRADO (HOSPEDERO) Y LAS CONDICIONES AMBIENTALES QUE DETERMINAN LA ACTIVACIÓN DEL COMPORTAMIENTO, ASÍ COMO LA DETECCIÓN Y ORIENTACIÓN HACIA EL POSIBLE HOSPEDERO. LAS CONDICIONES FISIOLÓGICAS DEL VECTOR DETERMINAN SU PREFERENCIA ALIMENTICIA, Y SU NECESIDAD Y DISPOSICIÓN PARA ALIMENTARSE. LAS CONDICIONES AMBIENTALES (TEMPERATURA, LUZ, HUMEDAD) INFLUYEN EN LA ACTIVACIÓN DEL COMPORTAMIENTO DE BÚSQUEDA, QUE SE VE ADEMÁS INFLUIDA POR FACTORES FísICOS (VISUALES). LA ASOCIACIÓN ENTRE FACTORES FíSICOS (VIENTO) Y QUímicos (OLORES) ORIENTAN AL MOSQUITO AL HOSPEDERO POR MEDIO DE ESTímULOS QUE EMANAN DEL MICROAMBIENTE DOMICILIAR Y PERIDOMICILIAR. A CORTA DISTANCIA, ESTÍMULOS QUÍMICOS DEL HOSPEDERO (COMPUESTOS POCO VOLÁTILES) INDUCEN EL ATERRIZAJE SOBRE EL HOSPEDERO Y FINALMENTE, LA PICADURA ES ESTIMULADA POR FACTORES QUímICOS (ÁCIDOS GRASOS) COMO POR FÍsICOS (HUMEDAD Y TEMPERATURA).

vuelo de los mosquitos hembra se oriente hacia el posible hospedero. Posteriormente, entran en juego otras sustancias poco volátiles, como son los olores expedidos en el sudor. Una vez que los mosquitos han aterrizado sobre el hospedero, estímulos de contacto como la temperatura y la humedad del cuerpo a través de higroreceptores y termorreceptores en las antenas son determinantes en la decisión de introducir o no sus probóscides $^{16}$ (figura 1 ).

El papel del $\mathrm{CO}_{2}$ en la atracción de mosquitos es el mejor documentado y ha sido estudiado, tanto en laboratorio como en campo en un número elevado de especies. ${ }^{17}$ Desde el primer reporte como atrayente de mosquitos, ${ }^{18}$ el $\mathrm{CO}_{2}$ se ha usado en trampas (con y sin luz ) para la captura y monitoreo de poblaciones de mosquitos silvestres. ${ }^{19}$

La distinción entre el efecto atrayente del $\mathrm{CO}_{2} \mathrm{y}$ el efecto estimulante de la temperatura y humedad corporal para la picadura fue reconocida desde $1963 .{ }^{20}$ Congruentemente, la remoción de $95.5 \%$ del $\mathrm{CO}_{2}$ expirado por un humano, redujo el número de mosquitos atraídos por este hospedero, mientras que la proporción de mosquitos que, ya atraídos, intentaban alimentarse sobre él fue la misma. ${ }^{21}$ Sin embargo, en experimentos del mismo tipo, la remoción del $\mathrm{CO}_{2}$ produjo una mayor reducción en la atracción de Culex que de Anopheles, lo que indica que las especies anofelinas probablemente usan, además, otros olores para la localización de sus hospederos. ${ }^{22}$

\section{Participación del octenol}

La participación de los olores expedidos por los humanos en la atracción de los vectores del paludismo en Africa fue reconocida desde $1942 .{ }^{23}$ Entre los compuestos que constituyen los olores de mamíferos se encuentra el 1-octen-3 ol (octenol). ${ }^{24}$ El efecto atrayente de este compuesto fue observado primero en Glossina spp..$^{25} \mathrm{Ala}$ fecha, se ha documentado que este compuesto atrae a una amplia gama de dípteros hematófagos incluyendo especies de Tabanidae, ${ }^{26}$ Oestridae, ${ }^{27}$ Stomoxyina $e^{28}$ y Ceratopogonidae. ${ }^{29}$ Un incremento en capturas de Aedes taeniorhynchus, Anopheles crucians, Ae quadrimaculatus y Wyeomyia mitchellii en trampas de luz 
CDC modificadas para realizar liberaciones del octenol fue la primera documentación del efecto atrayente de este alcohol sobre mosquitos. ${ }^{30}$ Actualmente, se ha documentado el efecto atrayente de este compuesto, solo o en combinación con otros, en 35 especies de mosquitos. ${ }^{31}$

\section{Participación del ácido láctico}

También han sido reportados como atrayentes de anofelinos otros compuestos presentes en emanaciones de animales y humanos como acetona, estradiol, cadaverina, lisina y ácido láctico. ${ }^{32} \mathrm{El}$ ácido láctico que es excretado con el sudor en la piel de los humanos es el principal atrayente de Ae aegypti ${ }^{33}$ y este compuesto también tiene efectos atrayentes sobre An albimanus. ${ }^{34}$ Recientemente, se reportó que hembras de An gambiae sensu lato y An funestus, los principales vectores del paludismo en el este de Africa, responden al L-ácido láctico, al ácido hexanoico y al $\mathrm{CO}_{2}$, solos o mezclados, y es la combinación del L-ácido láctico con $\mathrm{CO}_{2}$ la de mayor atracción..$^{35}$

El L-ácido láctico, en la piel de los humanos, es el producto final de la glicólisis durante el metabolismo anaeróbico de las células mioepiteliales de las glándulas sudoríparas ecrinas. ${ }^{36}$ Las glándulas ecrinas están presentes en todo el cuerpo de los humanos, juegan un papel importante en la termorregulación ${ }^{37} \mathrm{y}$, en contraste con las glándulas apocrinas, no están asociadas con los folículos pilosos. Las glándulas apocrinas por su parte se encuentran en gran número en las axilas, contienen a menudo células mioepiteliales y participan en la excreción de sudor y, por consiguiente, en la termorregulación de algunos animales de gran tamaño. ${ }^{38-40}$ El contenido de L-ácido láctico en la piel de humanos es mayor que en otros mamíferos ${ }^{41} \mathrm{y}$ las diferencias en la atracción de los humanos sobre hembras de mosquitos está asociada con las diferencias en los niveles del L-ácido láctico..$^{39,42}$ Estos niveles están relacionados con la densidad de glándulas sudoríparas ecrinas presentes, su actividad y diferencias de $\mathrm{pH}^{43}$

\section{Mosquitos zoofílicos y antropofílicos}

Las diferencias entre los compuestos liberados con la transpiración de humanos y animales son detectadas por los mosquitos y constituyen el principal determinante de la preferencia alimenticia. Así, los mosquitos zoofílicos como An quadriannulatus se orientan por un atrayente generalista como lo es el $\mathrm{CO}_{2}$, mientras que los mosquitos antropofílicos son atraídos por olores específicos del humano. ${ }^{44,45}$
La distribución de estos olores en el cuerpo parece dirigir a los mosquitos hacia el sitio de picadura. Mientras que otras especies más generalistas prefieren picar en partes de la cara, An gambiae prefiere picar en los pies y piernas. ${ }^{46,47}$ Interesantemente, estos mosquitos son atraídos por el queso Limburger ${ }^{48}$ y se ha documentado que la exposición de mosquitos a una mezcla de 12 de los más abundantes ácidos grasos presentes en este queso ocasiona una respuesta electrofisiológica y conductual dependiente de la dosis. ${ }^{49,50} \mathrm{La}$ capacidad de los mosquitos para responder a estos compuestos parece deberse a una similitud odorífera. Las hendiduras interdigitales de los pies de humanos suelen estar colonizadas por una bacteria, Brevibacterium epidermidis, un pariente cercano del Brevibacterium linens, que participa en los procesos fermentativos en la producción del queso en cuestión. La presencia de estas bacterias durante la manufactura del queso, explica su atracción por mosquitos que lo reconocen como una parte del cuerpo humano por la que tienen una preferencia especial. ${ }^{51}$ La aplicación práctica de este hallazgo sugiere la utilización de trampas que utilicen como cebo el queso, como se ha hecho antes para atrapar ratones. ${ }^{52}$ El efecto atrayente de los ácidos grasos emanados de la piel de humanos también ha sido observado para el Ae aegypti, una especie netamente antropofílica. ${ }^{53}$

Los ácidos grasos y la microflora de la piel

Los ácidos grasos constituyen la cuarta parte de la superficie de la piel de los humanos. Estos ácidos son producidos por la rotura de triglicéridos a glicerol libre por la acción de microrganismos, como Corynebacterium y Malessezia (Pityrosporum), presentes en las glándulas sebáceas, ${ }^{54,55}$ la cual es influida por el $\mathrm{pH}$ de la piel. Como estas condiciones varían entre individuos, se ha propuesto como la causa de los diferentes niveles de atracción entre humanos para An gambiae. . $6,57^{-}$

Los factores que determinan las variaciones en la microflora de la piel humana y su efecto sobre las interacciones mosquito-hospedero son aún desconocidos. Aunque las investigaciones en este campo aún están en sus inicios, es posible que, entre otros factores, las actividades laborales, los hábitos de alimentación e higiene personal sean causas de variaciones en la abundancia y metabolismo de la microflora. Estudios en el laboratorio han proporcionado inferencias acerca del efecto de las condiciones higiénicas de los individuos sobre su atracción por los mosquitos. En experimentos con hembras de An gambiae sensu stricto, éstas fueron atraídas por sudor incubado durante más de un día, en tanto que ninguna respuesta conductual y elec- 
trofisiológica se observó cuando se les expuso a sudor fresco (recién colectado). ${ }^{58}$ Los análisis cromatográficos de ambos sudores revelaron que los compuestos más abundantes en el sudor incubado fueron indol (27.9 \%), 1-dodecanol (22.4\%) y 3-metil-1-butanol (10 $\%)$. Estos compuestos no están presentes en el sudor fresco. ${ }^{59}$

\section{Efecto del $p H$}

Se han observado diferencias en la atracción sobre $A n$ gambiae ss. en voluntarios cuyo sudor difiere en el $\mathrm{pH}$. Los voluntarios que más atrajeron a estos mosquitos producían un sudor alcalino ( $\mathrm{pH} 8$ ), mientras que quienes indujeron pobre respuesta producían un sudor ácido ( $\mathrm{pH}$ 5.5). ${ }^{60} \mathrm{La}$ acidez del sudor fresco se debe a la producción y secreción de ácido láctico por las glándulas ecrinas, ${ }^{61}$ mientras que el cambio a $\mathrm{pH}$ alcalino en el sudor añejo es causado por la formación de amonio, debido a bacterias presentes en la piel. ${ }^{62}$ Estas observaciones contribuyen a explicar las preferencias alimenticias entre géneros y especies de mosquitos con preferencia por humanos. ${ }^{63,64}$

\section{Otros compuestos atrayentes}

Entre 300 y 400 compuestos se han identificado a partir de emanaciones del cuerpo de los humanos, y las cantidades producidas por éstos varían de una persona a otra. ${ }^{49}$ En extractos dietílicos de sudor se identificaron 73 compuestos de los cuales 40 fueron ácidos carboxílicos. Entre ellos, se observó respuesta de hembras de An gambiae a los ácidos 2-oxopentanoico, 2-oxobutanoico, 2-oxo-3-metilbutanoico, 2-oxo-3-metilpentanoico, 2-oxo-4-metilpentanoico y 2-oxohexanoico. ${ }^{65,66}$ También se ha observado respuesta conductual y antenal de este mosquito a los ácidos (E)- y (Z)-3-metil-2hexanoico y al 7-octenoico. La respuesta fisiológica fue similar a la reportada con algunos estimulantes como el 1-octen-3-ol, p-cresol y al ácido isovalerico; pero menor a la inducida con L-ácido láctico y al repelente sintético $\mathrm{N}, \mathrm{N}$-dietiltoluamida (DEET). ${ }^{67} \mathrm{El}$ gran número de compuestos volátiles con atracción diferencial sobre diferentes especies de mosquitos vectores de enfermedades ha hecho posible el considerar la posibilidad de desarrollar un olor sintético man in a bottle para atraer y capturar mosquitos cuando éstos están en busca de hospederos. ${ }^{68}$ Esta herramienta podría ser usada para el monitoreo de poblaciones de mosquitos en programas de vigilancia epidemiológica, o en combinación con insecticidas para incrementar su efectividad en programas de control de vectores.

\section{Efecto de la infección}

Existen otros factores relacionados con el comportamiento de los mosquitos que favorecen la transmisión de patógenos, pero que todavía no están bien entendidos. En An gambiae las hembras infectadas con los parásitos causantes del paludismo (Plasmodium) se alimentan más veces y absorben mayores cantidades de sangre que las hembras no infectadas. Esto mismo ocurre con An gambiae sl y An funestus infectados, los cuales intentan picar más veces sobre hospederos experimentales que los mosquitos no infectados. ${ }^{69,70}$ Por otro lado, la condición del hospedero también puede favorecer la transmisión del patógeno; así, se ha documentado que An stephensi se alimentan más en ratones con elevados números de las formas infectivas (gametos) de Plasmodium que en ratones no infectados. Es posible que en este último caso los comportamientos de defensa contra los mosquitos de los hospederos estén reducidos por la parasitosis. ${ }^{71}$ Sin embargo, también es posible que la mayor atracción por hospederos infectados con las formas infectivas del parásito pueda estar mediada por señales olfatorias, aún no identificadas, inducidas en el hospedero vertebrado por el parásito. ${ }^{72,73}$ De cualquier modo, es obvio que esta situación le confiere al parásito una fuerte ventaja selectiva. $^{74}$

\section{Un ecosistema de olores}

La integración de señales de atracción físico-químicas y la diversidad de éstas en los ambientes domésticos es probablemente responsable de la gran variabilidad en la atracción de mosquitos en las casas de localidades endémicas. Esto conduce a una focalización en la transmisión, y resulta en la recurrencia de casos palúdicos en pocas casas, mientras que otras se encuentran libres de la enfermedad. Los resultados de algunos estudios indican que las casas habitadas por humanos atraen significativamente más An gambiae y An funestus que las casas desocupadas, con una correlación positiva entre el número de habitantes y el número de mosquitos capturados. ${ }^{75,76}$ Sin embargo, además de los atrayentes individuales ya mencionados, los olores presentes en las casas que pueden tener influencia en la orientación de los mosquitos hacia ellas no han sido estudiados. Recientemente, se han identificado diversos factores asociados a casas en donde ocurren más casos de paludismo en el sur de México, entre ellos se encuentran el número de individuos, la limpieza dentro y fuera de la casa, la higiene personal (frecuencia del baño y lavado de ropa), entre otros (J Méndez, Di- 
rección General de Control de Vectores, Secretaría de Salud. Comunicación personal).

\section{Perspectivas}

Los factores químicos que influyen en la selección de los hospederos por los mosquitos vectores de enfermedades están siendo estudiados con la finalidad de crear nuevos métodos funcionales de muestreo y para el desarrollo de alternativas de control. El estudio de los atrayentes asociados a estas condiciones de vivienda podría aportar información para el diseño de estrategias que puedan ser dirigidas al control en casas de alto riesgo.

\section{Aplicación en monitoreo y control de vectores}

El creciente aumento en el número de especies y poblaciones de mosquitos resistentes a insecticidas ${ }^{77-80}$ incrementa permanentemente los costos y las dificultades para mantener un control adecuado y sostenido. La identificación de atrayentes químicos de mosquitos de importancia médica abre nuevas posibilidades para dirigir y focalizar la aplicación de insecticidas, por medio del manipuleo del comportamiento de búsqueda de hospedero, usando trampas con estos atrayentes. Por otra parte, el conocimiento de las señales químicas que intervienen en la selección del hospedero y sus orígenes podría usarse para dirigir las medidas de control hacia sitios específicos. Esta información también puede ser útil para la aplicación de intervenciones destinadas a eliminar las variables que ocasionan los olores atrayentes, como serían el cambio de hábitos alimenticios y promover la higiene del individuo y de sus viviendas.

La transmisión del paludismo en México presenta una focalización de casos en una porción baja de casas dentro de una localidad, además de una recurrencia de la enfermedad en unos pocos individuos. Esta situación confirma observaciones en otras áreas palúdicas e indica la pertinencia de investigar los factores propios de las casas y de los individuos que atraen a los mosquitos. ${ }^{81}$ Los estudios para conocer los aspectos químico-ecológicos de los vectores del paludismo en nuestro país son escasos y aislados. Las investigaciones para determinar la preferencia y disponibilidad de hospederos, estimando los índices de forrajeo y preferencia alimenticia, ${ }^{2,82}$ deben ser reexaminados para investigar y evaluar si existen grados de antropofiliazoofilia. La existencia de grados variables de preferencia alimenticia podría conducir a que una parte de la población de mosquitos, con inclinaciones antropofíli- cas, ingresara preferentemente a las casas en busca de alimento. Esto explicaría la focalización de la transmisión a unas cuantas casas de las localidades. Esta información serviría para complementar las estrategias de control actualmente empleadas.

\section{Transgénesis para el control de vectores}

Los avances en biología molecular que permite la transformación genética estable de mosquitos ${ }^{83}$ y la reciente publicación del genoma completo de An gambiae ${ }^{84}$ abren, a su vez nuevas posibilidades para el desarrollo de estrategias de control basadas en la ingeniería genética de los vectores. ${ }^{85}$ En esfuerzos previos, estrategias de manipulación genética de los vectores estuvieron dirigidas a la producción y liberación de mosquitos (machos) estériles. Puesto que los mosquitos hembra copulan una sola vez y almacenan el esperma para ser usado en todos sus lotes de huevos, la cópula con machos estériles condena a la hembra a no tener descendencia. Sin embargo, esta estrategia fue poco efectiva debido a la necesidad de producir y liberar de manera permanente grandes números de mosquitos estériles. ${ }^{86}$ Actualmente, las estrategias de manipulación genética están dirigidas no a evitar la reproducción de los mosquitos, sino a la introducción de genes nuevos o modificados en las poblaciones silvestres. Estos genes pueden codificar compuestos tóxicos para el parásito, con lo cual los mosquitos no se infectarían y no podrían transmitir la enfermedad. ${ }^{87,88}$

La identificación de receptores químicos en las antenas de los mosquitos ${ }^{89,90}$ y las señales que hacen que éstos respondan a atrayentes de animales son la base para la identificación de los genes involucrados. Cuando éstos sean identificados, será posible manipularlos para cambiar su preferencia alimenticia y desviar la picadura de mosquitos actualmente antropofílicos hacia otros animales. Aunque estos estudios tienen interesantes avances es importante resaltar que su incorporación en estrategias de control será en un largo plazo.

Obedeciendo a la magnitud del problema del paludismo en Africa, el complejo de An gambiae ocupa el centro de la atención de investigaciones sobre los factores que influyen en selección de sus hospederos. Aunque existen atrayentes comunes, también existe una gran variabilidad en las características de los vectores y en su respuesta a una gran diversidad de señales físico químicas, lo cual obliga al estudio de los vectores locales para poder hacer uso de las nuevas tecnologías, con un diseño ex profeso para nuestras condiciones y mosquitos locales. 


\section{Referencias}

1. D avis EE. Introduction II. O Ifactory control of mosquito behaviour. En: Bock GR, Cardew G, Ed. 0 Ifaction in mosquitoes-host interactions. Ciba Fundation Symposium 200. Chichester: John W iley \& Sons, 1996:48-54.

2. Loyola EG, González-Cerón L, Rodríguez-López MH,ArredondoJiménez JI, Bennett S, Bown DN . Anopheles albimanus (Diptera:Culicidae) host selection patterns in three ecological areas of the coastal plains of Chiapas, southern Mexico. J Med Entomol 1993;30:18-23.

3. Rubio-Palis Y, C urtis CF, G onzález C,W irtz RA. Host choice of anopheline mosquitoes in a malaria endemic area of western Venezuela. Med Vet Entomol 1994;8:275-280.

4. D ixit V, Gupta AK, Kataria O M, Prasad GB. Host preference of Culex quinquefasciatus in Raipur city of Chattisgarh State. J Commun D is 2001;33:17-22.

5. Ree HI, Hwang UW, Lee IY, Kim TE. Daily survival and human blood index of Anopheles sinensis, the vector species of malaria in Korea. J Am Mosq Control Assoc 2001;17:67-72.

6. A merasinghe $\mathrm{PH}, \mathrm{Amerasinghe} \mathrm{FP.} \mathrm{Multiple} \mathrm{host} \mathrm{feeding} \mathrm{in} \mathrm{field}$ populations of Anopheles culicifacies and An subpictus in Sri Lanka. Med Vet Entomol 1999;13:124-131.

7. Dye $C$. The analysis of parasite transmission by blood-sucking insects. Ann Rev Entomol 1992;37:1-19.

8. Panday RS. Anopheles nuneztovari and malaria transmission in Surinam. Mosq N ews 1977;37:728-738.

9. Charlwood JD, Alecrim W A. C apture-recapture studies with the South American malaria vector Anopheles darlingi Root.Ann Trop Med Parasitol 1989;83(6):569-576.

10. Kakitani I, Forattini O P. Paridade e desenvolvimento ovariano de Anopheles albitarsis sl em área de agro-ecossistema irrigado. Rev Saude Publ 2000;34:33-38.

11. Cork A. O lfactory basis of host location by mosquitoes and other haemato phagous Diptera. En: Bock GR, Cardew G, Ed. O Ifaction in mosquitoes-host interactions. Ciba Fundation Symposium 200.

Chichester: John W iley \& Sons, 1996:71-88.

12. Allan SA, D ay JF, Edman JD.Visual ecology of biting flies. Ann Rev Entomol 1987;32:297-316.

13. Bidlingmayer W L. How mosquitoes see traps: Role of visual responses. J Am Mosq Control Assoc 1994;10:272-279.

14.Takken W. The role of olfaction in host seeking at mosquitoes:A review. Insect Sci Appl 1991;12:287-295.

15. Klowden MJ. Endogenous factors regulating mosquito host-seeking behaviour: En: Bock GR, Cardew G, Ed. 0 Ifaction in mosquitoes-host interactions. Ciba Fundation Symposium 200. Chichester: John W iley \& Sons, 1996:212-225.

16. Davis EE, Sokolove PG. Temperature responses of antennal receptors of the mosquito Aedes aegypti. J Comp Physiol A 1975;96: 223-236.

17. Takken W, Knols BG. 0 dor-mediated behavior of A frotropical malaria mosquitoes. Annu Rev Entomol 1999;44:131-157.

18. Rudolfs W. Chemetropism in mosquitoes. Bull N J Agr Exp Stn 1922;367:4-23.

19. Khan AA, Maibeach HI. Cuantitation of the effect of several stimuli on landing and prosing by Aedes aegyti. J Econ Entomol 1996;59:902-905. 20. Hocking B.The use of attractants and repellents in vector control. Bull W orld Health 0 rgan 1963;29(Suppl):121-126.

21. Snow W F.The affect of a reduction in expired carbon dioxide on the attractiveness of human subjects to mosquitoes. Bull Entomol Res 1970;60:43-48.
22. Constantini C, Gibson G, Sagnon N F, D ella Torre A, Brady J. Coluzzi M. Mosquitoes responses to carbon dioxide in W est African Sudan savanna village. Med Vet Entomol 1996;10:220-227.

23. D e Jong R, Knols BG. 0 Ifactory responses of host-seeking Anopheles gambiae ss. Giles (Diptera: Culicidae). Acta Trop 1995;59:333-335.

24.Takken W, D ekker T, W ijnholds YG 0 o dor mediated flight of Anopheles gambiae Giles sensu strictu and An. stephensi Liston in response to $\mathrm{CO} 2$, acetone, and 1-octen-3-ol.J Insect Behav 1997;10:395-407.

25.Vale GA.The response of tsetse flies (Diptera: Glossinidae) to ox odour. Bull Entomol Res 1974;64:541-588.

26. French FE, Kline DL. 1-octen-3-ol an effective attractant for Tabanidae. J Med Entomol 1989;26:459-461.

27. Anderson IH, Jaenson TG T. N ectar feeding by mosquitoes in Sweden, with special reference to Culex pipiens and Cx. torrentium. Med Vet Entomol 1987;1:59-64.

28. Holloway MTP, Phelps RJ.The response of Stomoxis spp (Diptera:M uscidae) to traps and artificial host odours in the field. Bull Entomol Res 1991;81:51-55.

29. Blackwell A, W adhams LJ, Mordue W. Electrophysiological and behavioural studies of the biting midge, Culicoides impunctatus Goetghebuer (Diptera:Ceratopogonidae): Interactions between some plant-derived repellent compounds and a host-odour attractant, 1-octen-3-ol. Physiol Entomol 1997; 22:102-108.

30.Takken W, Kline DL. Carbon dioxide and 1-octen-3-ol as mosquito attractant. J Am Mosq Control Assoc 1989;5:311-316.

31. Kline D L. O Ifactory attractants for mosquito surveillance and control: 1-octen-3-ol. J Am Mosq Control Assoc 1994;10:280-287. 32. Schreck CE, Smih N, Carlson DA, Price GD, Haile D, Godwin DR.A material isolated from human hands that attracts female mosquitoes. J Chem Ecol 1981;8:429-438.

33. Geier M, Sass H, Boeckh J.A search for components in human body odour that attract females of Aedes aegypti. En: Bock GR, C ardew G, Ed. 0 Ifaction in mosquitoes-host interactions. Ciba Fundation Symposium 200. C hichester: John W iley \& Sons, 1996;132-148.

34. Rodríguez-C otta E. Evaluación del efecto atrayente de 2-butanona, acetona y $L$-ácido láctico sobre la alimentación de hembras de Anopheles albimanus W iedemann (Diptera:Culicidae) en condiciones de laboratorio (Tesis).Tuxtla Gutiérrez, Chiapas: Universidad Autónoma de Chiapas. México, 2001.

35. Murphy MW, Dunton RF, Perich MJ, Rowley W A.Attraction of Anopheles (Diptera: Culicidae) to volatile chemicals in W estern Kenya. J Med Entomol 2001;38:242-244.

36.A cre F Jr,Turner RB, G ouck HK, Beroza MY, Smith N . L-lactic-acid:A mosquito attractant isolated from humans. Science 1968;161:

1346-1347.

37. Schreck CE, Kline DL, C arlson DA. Mosquito attraction to substances from the skin of different humans. J Am Mosq Control Assoc 1990;6:406-410.

38. Steib BM, G eier M, Boeckh J.The effect of lactic acid on odour-related host preference of yellow fever mosquitoes. Chem Senses 2001;26:523-528.

39. Robertshaw D.Apocrine sweat glands. In: Goldsmith LA, Ed.Physiology, biochemistry, and molecular biology of the skin. 0 xford: 0 xford University Press,1991;763-775.

40. So kolovVE. Mammal skin. Bekerley: University of California Press, 1982.

41.Allen TE, Blight J.A comparative study of the temporal patterns of cutaneus water vapour loss from some domesticated mammals, with epitrichial sweat glands. Comp Biochem Physiol 1969;31:347-363. 42. Jenkinson D MCE, Montgomery I, Elder HY.The ultrastructure of the sweat glands of the $0 x$, sheep and goat during sweating and recovery. J Anat 1979;129:117-140. 
43. D ekker T, Steib B, C arde RT, G eier M. L-lactic acid:A humansignifying host cue for the anthropophilic mosquito Anopheles gambiae. Med Vet Ento mol 2002;16:91-98.

44. Bosch 0 J, G eier M, Boeckh J. Contribution of fatty acids to olfactory host finding of female Aedes aegypti. Chem Senses 2000;25:323-330. 45. D ekker T, Takken W, Braks MA. Innate preference for host-odor blends modulates degree of anthropophagy of Anopheles gambiae sensu lato (Diptera: Culicidae). J Med Entomol 2001;38:868-871.

46. De Jong R, Knols BGJ. Selection of biting sites on man by two malaria mosquito species. Experientia 1995;51:80-84.

47. Dekker T,Takken W, Knols BG J, Bouman E,Van der Laak S et al. Selection of biting sites on a man host by Anopheles gambiae sensu strictu, An. arabiensis and An quadriannulatus. Entomol Exp Appl 1998:87:295-300

48. Knols BGJ, De Jong R. Limburger cheese as an attractant for the malaria mosquito Anopheles gambiae ss. Parasitol Today 1996;12:159-161. 49. Cork A, Park KC. Identification of electrophysiologically-active compounds for the malaria mosquito, Anopheles gambiae in human sweat extracts. Med Vet Entomol 1996;10:269-276.

50. Knols BG J,Van Loon JJA, Cork A, Robinson RD, Adan W et al. Behavioral and electrophysiological responses of the female malaria mosquito Anopheles gambiae (Diptera:Culicidae) to Limburger cheese volatiles. Bull Entomol Res 1997;87:151-159.

51. De Jong R, Knols BGJ. Selection of biting sites by mosquitoes. En: Bock $G R, C$ ardew $G$, Ed. 0 Ifaction in mosquitoes-host interactions. Ciba Fundation Symposium 200. Chichester: John W iley \& Sons, 1996:89-103

52. Knols BGJ. On human odour, malaria mosquitoes, and Limburger cheese. Lancet 1996;348:1322.

53. Carlson DA, Smith N, Gouck HK, Godwin D R. Yellow fever mosquitoes: Compounds related to lactic acid that attract females. J Econ Entomol 1973;66:329-331.

54. N icolaides N . Skin Lipids:Their biochemical uniqueness. Science 1974;186:19-26.

55. Puhvel SM, Reisner RM, Sakamoto M. A nalysis of lipid composition of isolated human sebaceous gland homogenates after incubation with cutaneous bacteria:Thin layer chromatographic. J Invest Dermatol 1975;64:406-411

56. Knols BGJ. 0 dours-mediated host-seeking behavior of the Afro-tropical malaria vector Anopheles gambiae Giles (Ph.D. thesis). $W$ ageningen The $N$ etherlands: $W$ ageningen A gricultural U niver sity. 1996:213.

57. Knols BG J, Takken W, Charlwood D, De Jong R. Species-specific attraction of Anopheles mosquitoes (Diptera:Culicidae) to different humans in south east-Tanzania. Proc Exp Appl Entomol 1995;6:201-206. 58. Braks MAH, Takken W. Incubated human sweat but not fresh sweat attracts the malaria mosquito Anopheles gambiae sensu stricto.J Chem Ecol 1999;25:663-672.

59. Meijerink J, Braks MAH, Brack AA, Adam W, D ekker T, Posthumus MA et al. Identification of olfactory stimulants for Anopheles gambiae from human sweat samples.J Chem Ecol 2000;26:1367-1382. 60. Braks MAH, Cork A,Takken W. O Ifactometer studies on the attraction of Anopheles gambiae sensu stricto (Diptera:Culicidae) to human sweat. Proc Exp Appl Entomol 1997:8:99-104.

61.Thurmon FM, 0 ttenstein B. Studies on the chemistry of human perspiration with especial reference to its lactic acid content.J Invest Dermatol 1952;18:333-339.

62. Bergeim 0,C ornbleet T.The antibacterial action of the lactic acid and volatile fatty acids of sweat. Am J Med Sci 1943;206:785-792. 63. Smith CN, Smith N, Gouck HK, W eidhaas DE, G ilbert IH, Mayer M et al. L-lactic acid as a factor in the attraction of Aedes aegypti (Diptera: Culicidae) to human hosts. Ann Entomol Soc Am 1970;63:760-770.
64. Bernier UR, Kline DL, Schreck CE,Yost RA, Barnard D R. Chemical analysis of human skin emanations: $C$ omparison of volatiles from humans that differ in attraction of Aedes aegypti (Diptera:Culicidae). J Am Mosq Control Assoc 2002;18:186-195.

65. Healy TP, Copland MJ. Human sweat and 2-oxopentanoic acid elicit a landing response from Anopheles gambiae. Med Vet Entomol 2000;14:195-200.

66. Healy TP, C opland MJ, C ork A, Przyborowska A, Halket JM. Landing responses of Anopheles gambiae elicited by oxocarboxylic acids. Med Vet Entomol 2002:16:126-132.

67. Constantini C, Birkett MA, G ibson G, Ziesmann J, Sagnon N F, Mohammed $\mathrm{HA}$ et al Electroantennogram and behavioural responses of the malaria vector Anopheles gambiae to human-specific sweat components. Med Vet Entomol 2001;15:259-266.

68. Takken W, Knols BG J. 0 dor-mediated behaviour of afrotropical malaria mosquitoes. Ann Rev Entomol 1999:44:131-157.

69. Koella JC, Sorensen FL,Anderson RA.The malaria parasite, Plamosdium falciparum increases the frequency of multiple feeding of its mosquitoes vector Anopheles gambiae. Proc Roy Soc London Ser B $1998: 265: 763-768$

70. W ekesa JW, C opel RS, Mwangi RW. Effect of Plasmodium falciparum on blood feeding behavior of naturally infected Anopheles mosquitoes in western Kenya. Am J Trop Med Hyg 1992;47:484-488.

71. Edman JD, Kale HW. Host behaviour: Its influence on the feeding success of mosquitoes. Ann Entomol Soc Am 1971;64:513-516. 72. D ay JF, Edman JD. Malaria renders mice susceptible to mosquito feeding when gametocytes are most infective. J Parasitol 1983;69: 163-170.

73. D ay JF, Edman JD.The importance of disease induced changes in mammalian body temperature to mosquito blood feeding. Com Biochem Physiol 1984;77A:447-452.

74. Hurd H. Physiological and behavioural interactions between parasites and invertebrate hosts. Adv Parasitol 1990;29:271-317. 75. Guilles MT,W ilkes TJ.A comparison of the range of attraction of animal baits and carbon dioxide for some W est African mosquitoes. Bull Entomol Res 1968;59:441-456.

76. Guilles MT,W ilkes TJ.The range of attraction of single baits for some W est African mosquitoes. Bull Entomol Res 1970;60:225-235.

77. Georghiou GP,A riaratnam V, Breeland SG. D evelopment of resistance to carbamates and organophosphorus compounds in Anopheles albimanus in nature. Bull W HO 1972;46:551-554.

78. Penilla RP, Rodríguez AD, Hemingway J,Torres JL,A rredondo-Jiménez $J I$, Rodríguez MH. Resistance management strategies in malaria vector mosquito control. Baseline data for a large-scale field trial against Anopheles albimanus in Mexico. Med Vet Entomol 1998;12:217-233. 79. Ranson H, Claudianos C, O rtelli F,A bgrall C, Hemingway J, Sharakhova MV et al. Evolution of supergene families associated with insecticide resistance. Science 2002;298:179-181.

80. Hemingway J, Field L,Vontas J.An over view of insecticide resistance. Science 2002;298:96-97.

81.Yadav RS, G hosh SK. Radical curative efficacy of five-day regimen of primaquine for treatment of Plasmodium vivax malaria in India. J Parasitol 2002;88:1042-1044

82. Fernández-Salas I, Roberts DR, Rodríguez MH, Rodríguez MC, Marina-Fernández CF. Host selection patterns of Anopheles pseudopunctipennis under insecticide spraying situations in southern Mexico. J Am Mosq Control Assoc 1993;9:375-384.

83. Ito J, G hosh A, Moreira LA,W immer EA, Jacobs-Lorena M.Transgenic anopheline mosquitoes impaired in transmission of a malaria parasite. $N$ ature 2002:417:452-455.

84. Holt RA, Subramanian GM, Halpern A, Sutton G G, Charlab R, $N$ usskern DR, W incker $P$ et al.The genome sequence of the malaria mosquito Anopheles gambiae. Science 2002;298:129-149 
85. Carlson J0. Genetic manipulation of mosquitoes: An approach to controlling disease. Trends Biotechnol 1996;14:447-448.

86. Curtis CF, Townson H. Malaria: Existing methods of vector control and molecular entomology. Br Med Bull 1998;54:311-325.

87. James AA, Beerntsen BT, Capurro ML, Coates C J, Coleman J, Jasinskiene $\mathrm{N}$ et al. Controlling malaria transmission with geneticallyengineered, Plasmodium-resistant mosquitoes: Milestones in a model system. Parassitologia 1999;41:461-471

88. Moreira LA, Ito J, G hosh A, D evenport M, Zieler H, A braham EG et al. Bee venom phospholipase inhibits malaria parasite development in transgenic mosquitoes. J Biol Chem 2002;277:40839-40843.
89. Hill CA, Fox AN, Pitts RJ, Kent LB, Tan PL, Chrystal MA et al. G protein-coupled receptors in Anopheles gambiae. Science 2002;298: 176-178.

90. Fox AN , Pitts RJ, Z wiebel LJ.A cluster of candidate odorant receptors from the malaria vector mosquito, Anopheles gambiae. $C$ hem Senses 2002;27(5):453-459. 